\title{
Comparison Of Nutrition And Quality Of Life Of Esophagogastrostomy And The Double-Tract Reconstruction After Laparoscopic Proximal Gastrectomy
}

\section{Bang Wool Eom}

National Cancer Center, Korea

Ji Yeon Park ( $\square$ jybark990118@naver.com )

Kyungpook National University Chilgok Hospital https://orcid.org/0000-0002-6178-7906

Ki Bum Park

Kyungpook National University School of Medicine

Hong Man Yoon

National Cancer Center, Korea

Oh Kyoung Kwon

Kyungpook National University School of Medicine

\section{Keun Won Ryu}

National Cancer Center, Korea

\section{Young-Woo Kim}

National Cancer Center, Korea

\section{Research}

Keywords: gastric cancer, proximal gastrectomy, esophagogastrostomy, double-tract reconstruction, quality of life

Posted Date: July 20th, 2020

DOI: https://doi.org/10.21203/rs.3.rs-42712/v1

License: (9) This work is licensed under a Creative Commons Attribution 4.0 International License. Read Full License

Version of Record: A version of this preprint was published at Medicine on April 16th, 2021. See the published version at https://doi.org/10.1097/MD.0000000000025453. 


\section{Abstract}

Purpose: This study aims to compare the nutritional outcomes and quality of life between patients who underwent esophagogastrostomy (EG) and those who underwent the double-tract reconstruction (DTR) after laparoscopic proximal gastrectomy for early gastric cancer.

Methods: We retrospectively reviewed the prospectively established database of 45 patients who underwent EG with anti-reflux procedure and 58 patients who underwent the DTR after laparoscopic proximal gastrectomy between December 2013 and June 2017. Then, we compared the baseline characteristics, clinical outcomes, postoperative nutritional parameters, and quality of life (QOL) using EORTC QLQ STO-22 between the EG and DTR groups.

Results: In the postoperative 1-year endoscopic findings, the incidence of esophageal reflux was higher in the EG group ( $17.8 \%$ vs. $3.4 \%, p=0.041)$ and there was no significant difference in anastomotic stricture. Nutritional status was assessed via body mass index, serum albumin, protein, hemoglobin, and ferritin; we found no significant differences. The incidences of iron deficiency anemia and vitamin B12 deficiency also showed no significant difference between the two groups. With regards to the quality of life, the difference values between preoperative and postoperative 1-year were evaluated; there was no significant difference between the EG with anti-reflux procedure and DTR groups.

Conclusions: EG had higher incidence of esophageal reflux and similar nutritional outcomes and QOL compared with the double-tract reconstruction after laparoscopic proximal gastrectomy. Additional largescale research is needed to evaluate the long-term functional outcomes of EG and the double-tract reconstruction.

\section{Introduction}

In recent decades, the worldwide incidence of proximal gastric cancers has increased steadily.[1-3] In cases where the proximal gastric cancer was resectable, either total or proximal gastrectomy has been performed. Theoretically, proximal gastrectomy had advantages in nutritional and functional aspects. However, high incidences of reflux esophagitis and anastomotic stenosis were problematic in proximal gastrectomy and the recurrence rate was also higher in proximal gastrectomy compared with total gastrectomy. Total gastrectomy has become a standard procedure currently performed for the treatment of proximal gastric cancer. [4]

On the other hand, the incidence of early gastric cancer has increased due to the nationwide mass screening program in East Asia, especially in Korea and Japan.[3, 5, 6] Early gastric cancer has an excellent prognosis, and surgeons questioned whether total gastrectomy for small-sized early proximal gastric cancer is the best treatment option. While total gastrectomy has a lower risk of reflux esophagitis and anastomotic stenosis compared with proximal gastrectomy, it also leads to severe weight loss and long-term nutritional deficiencies. Thus, surgeons reconsidered proximal gastrectomy as the treatment for early proximal gastric cancer and tried to overcome the anastomotic complications by additional anti- 
reflux procedures or structural modification such as jejunal interposition and double-tract reconstruction (DTR).[7-11]

After proximal gastrectomy, there are two types of reconstruction methods according to the organ connected to the esophagus; esophagogastrostomy (EG) and esophagojejunostomy. Since conventional EG has a high risk of anastomotic complications, several new techniques were developed by adding preceding anti-reflux procedures.[7-9] In the other approaches using EG, including jejunal interposition and the double-tract reconstruction, the esophagus is connected to the jejunum, which prevents reflux of gastric acid directly into the esophagus.[10,11] Many studies have evaluated the feasibility and technical safety of these novel procedures and demonstrated the favorable results of proximal gastrectomy. In particular, nutritional outcomes were better in proximal gastrectomy compared with total gastrectomy. [8, $12,13]$ However, few studies have compared functional outcomes between the two types of reconstruction (EG vs. esophagojejunostomy) after laparoscopic proximal gastrectomy.

Therefore, in this study, we aim to compare the clinical and nutritional outcomes and quality of life between patients who underwent EG and those who underwent the double-tract method after laparoscopic proximal gastrectomy for early gastric cancer.

\section{Methods}

\section{Patients}

We retrospectively reviewed the prospective database of patients who underwent laparoscopic proximal gastrectomy for clinical stage I gastric cancer located in the upper one-third of the stomach from two large-volume hospitals in Korea. From December 2013 to June 2017, a total of 67 and 50 patients underwent laparoscopic proximal gastrectomy in the National Cancer Center and the Kyungpook National University Chilgok Hospital, respectively. Among them, we excluded 14 patients who were diagnosed as stage II or more in the pathological reports, and 103 patients were finally included in the analysis. No patients included in this study received adjuvant chemotherapy after operation.

The Institutional Review Board of the National Cancer Center (NCC2017-0120) and the Kyungpook National University Chilgok Hospital (2019-07-004) approved this study and waived the need for patient informed consent due to the retrospective nature of the study.

\section{Surgical procedure}

In the laparoscopic proximal gastrectomy, five working ports were inserted into the umbilicus $(12 \mathrm{~mm})$, right upper quadrant $(5 \mathrm{~mm})$, right lower quadrant $(12 \mathrm{~mm})$, left upper quadrant $(5 \mathrm{~mm})$, and left lower quadrant (5mm). D1+ lymph node dissection was performed according to the Japanese gastric cancer treatment guidelines, including lymph node stations 1, 2, 3a, 4sa, 4sb, 7, 8a, 9, and 11p. The right gastroepiploic artery and right gastric artery were saved to preserve blood supply to the remnant distal stomach. The hepatic branch of the vagus nerve was also preserved to maintain pyloric function. After 
confirming the tumor-negative resection margins in the frozen examination, reconstruction was performed.

In EG, we had two types of anti-reflux procedures in consecutive order. In the initial period (2013-2016), anchoring the gastric wall to the diaphragm was added to the conventional EG to create a neo-His angle and fundus. In the last period (2017), two interrupted sutures were performed to make an artificial His angle between the distal part of the esophagus's posterior wall and the proximal portion of the stomach's anterior wall. Then, the esophageal stump and stomach wall were opened, and anastomosis was performed with continuous hand-sewn sutures between the esophagus and stomach.

In the double-tract reconstruction, the linear-stapled jejuno-jejunal and gastrojejunal anastomoses were performed extracorporeally via mini-laparotomy. Then, intracorporeal esophagojejunostomy was performed using an endo-linear stapler via the overlap method.[14] The distances between the esophagojejunostomy and both the gastrojejunostomy and the jejunojejunostomy were estimated at 15 $\mathrm{cm}$ and $20 \mathrm{~cm}$, respectively.

\section{Baseline data collection}

We reviewed medical records to collect data on patient demographics and pathological characteristics. Comorbidity was evaluated using the American Society of Anesthesiologists (ASA) physical status classification system, and histological types were classified according to the 2010 World Health Organization (WHO) classification $[15,16]$. When a tumor consisted of two or more histological types, the quantitative predominance was recorded as the histological type. The pathological stage was categorized according to the $8^{\text {th }}$ American Joint Committee on Tumor-Node-Metastasis (TNM) classification system [17].

\section{Outcomes assessment}

Patients followed up every 6 months after surgery for 5 years. Each follow-up visit included measurement of body weight, laboratory tests, and a quality of life (QOL) assessment. Esophagogastroduodenoscopy was performed annually following surgery.

Laboratory tests included complete blood cell count, blood chemistry, and liver function testing. Serum ferritin, iron, and vitamin B12 levels have been checked periodically since 2015 and are tested annually to monitor for the development of iron deficiency anemia and vitamin B12 deficiency. Anemia was defined as a hemoglobin level $<13 \mathrm{~g} / \mathrm{dL}$ in men and $<12 \mathrm{~g} / \mathrm{dL}$ in women, based on World Health Organization criteria [18]. Iron deficiency was defined as a serum ferritin level $<30 \mathrm{ng} / \mathrm{mL}$, and vitamin B12 deficiency as a serum vitamin $B 12$ level $<200 \mathrm{pg} / \mathrm{mL}[19,20]$.

The presence of esophageal reflux was evaluated at the 1-year follow up via endoscopic examination, and the severity was classified according to the Los Angeles (LA) classification: LA grade A, one or several erosions limited to the mucosal folds and no larger than $5 \mathrm{~mm}$ in size; LA grade $B$, one or several 
erosions limited to the mucosal folds and larger than $5 \mathrm{~mm}$ in size; LA grade C, erosion extending over mucosal folds, but over less than three-quarters of the circumference; and LA grade $D$, confluent erosions extending over more than three-quarters of the circumference. [21]. Esophageal stricture usually occurs within several months after surgery, and patients experience dysphagia. We reviewed all endoscopic examination results performed postoperatively within 1-year and divided the patients into 3 groups: patients without any stricture, patients with mild stricture but no need for intervention, and patients who underwent endoscopic interventions such as balloon dilatation for severe stricture.

A QOL survey was performed in patients who visited the outpatient clinic for routine check-ups. QOL was assessed using the validated Korean version of the gastric cancer-specific module of the European Organization for Research and Treatment of Cancer (EORTC QLQ-STO22) [22, 23] The EORTC QLQ-STO22 is composed of 9 symptom scales, and each scale is represented by a score ranging from 0 to 100; a higher score indicates a poorer QOL.[24] The results of the QOL survey obtained preoperatively and 1-year postoperatively were included in the analyses.

\section{Statistical analysis}

The continuous variables are shown as the means \pm standard deviations or the medians with interquartile ranges, and the categorical variables are presented as proportions. Differences between two groups were tested using a t-test or the Wilcoxon rank-sum test for continuous variables and the one-way analysis of variance (ANOVA) or Kruskal-Wallis test was used for differences among three groups. The chi-square test or Fisher's exact test was used for categorical variables. A mixed-effect model was performed to analyze changes in the nutritional outcomes over time between the two groups.

In the QOL analysis, each subscale or item is presented as the median and the interquartile range. Nonparametric statistics (i.e., the Willcoxon rank-sum test) were used to evaluate their statistical significance because the distribution of QOL scores did not follow a normal distribution.

Data analyses were conducted using SAS version 9.4 (SAS Institute Inc., Cary, NC). P-values less than 0.05 were considered significant.

\section{Results}

\section{Patient characteristics}

In the patients' demographics, age, sex, and ASA classification showed no significant differences among the three groups, and body mass index (BMI) was highest in the EG group ( 25.0 vs. $23.5, p=0.018$ ) (Table 1). In pathological characteristics, the DTR group had the largest number of harvested lymph nodes (28.4 vs. 34.0; $p=0.022$ ). One and seven patients had tumor-invaded proper muscle (T2) in the EG without and with anti-reflux procedure groups, respectively, and there was no significant difference in the $\mathrm{N}$ classification.

\section{Anastomotic complications}


The presence of esophageal reflux was evaluated by endoscopic examination performed 1-year postoperatively. Incidence of esophageal reflux (LA classification B or C) was higher in the EG group than the DTR group ( $11.1 \%$ vs. $1.7 \%, \mathrm{p}=0.041)$ (Table 2$)$.

Incidence of severe anastomotic stricture seemed to be higher in the EG groups (11.1\% vs. $5.2 \%$ ), but not significant. $(p=0.068)$ All patients with severe anastomotic stricture underwent one or more balloon dilatations.

\section{Nutritional outcomes}

Nutritional parameter changes were evaluated, including body mass index (BMI), serum albumin, protein, hemoglobin, and ferritin in both groups (Fig 1). There was no significant difference in the changes in nutritional parameters between the EG and DTR groups ( $p=0.152,0.918,0.777,0.152$, and 0.450$)$.

Development of iron deficiency anemia and vitamin B12 deficiency were evaluated based on the laboratory findings of serum hemoglobin, ferritin, and vitamin B12 (Table 3). The percentage of iron deficiency anemia within postoperative year- 1 were $9.5 \%$ and $12.1 \%$ in the EG and DTR groups, respectively, and there was no significant difference $(p=0.757)$. There was no significant difference in the incidence of vitamin B12 deficiency either $(p=0.771)$.

\section{QOL outcomes}

There were considerable missing data, and finally 22 and 36 patients' QOL data were evaluated in this study. Table 4 shows differences between preoperative and postoperative 1-year values in each subscale of EORTC QLQ STO-22. Positive values were observed in all subscales, which indicate deterioration of QOL symptoms in postoperative year-1 compared with that in the preoperative period. Some differences were noted in reflux, anxiety, and body image ( 11.1 vs. $0,16.7$ vs. 0 , and 0 vs. 33.3 , respectively), but there was no significant difference in QOL changes between the two groups.

\section{Discussion}

In this study, we compared clinical and nutritional outcomes and QOL among the EG with and without anti-reflux procedure and DTR groups. EG and DTR have different structure in terms of food passage and some differences in nutritional outcomes and QOL were expected. However, we found that there was no significant difference in the clinical and nutritional outcomes and quality of life among the groups.

The advantage of EG is its resembling physiologic structure. Food passes through the stomach and duodenum and most nutrients, including iron and vitamin B12, can be absorbed into the duodenum and proximal jejunum after EG. Moreover, the conserved pyloric ring could reduce dumping syndrome, which might result in less body weight loss. Although the same distal part of the stomach is preserved in both the EG and DTR groups, the EG group is suggested to have better nutritional outcomes because all food does not pass through stomach and duodenum in the DTR group. Unlike this proposal, we found that EG is not superior with regards to nutritional parameters, even BMI. These results are similar with those of 
the study by Toyomasu et al.[8] which showed no significant difference in the blood tests, including serum hemoglobin, ferritin, albumin, and protein levels, between the gastric tube and jejunal interposition groups.

The disadvantage of EG is high incidence of postoperative reflux. To date, various anti-reflux procedures have been developed to reduce anastomotic complications and showed an acceptable incidence of anastomotic complications. $[7,9,25]$ In this study, we similarly added the performance of anti-reflux techniques such as a tagging suture between the stomach and diaphragm or fixation between the stomach and esophageal stump to make an artificial His angle. However, the EG group still had higher incidence of postoperative esophageal reflux compared to the DTR group. Therefore, further advanced procedures such as fundoplication and double flap method could be considered rather than simple tagging or fixation in the future.[7, 9]

In Korea, the double-tract reconstruction is the most common reconstruction method after proximal gastrectomy to avoid anastomotic complications.[3] The double-tract reconstruction is suggested to have some nutritional advantages compared to total gastrectomy because the distal part of the stomach remains. In previous studies, the double-tract reconstruction had better outcomes in laboratory findings related to iron deficiency anemia and vitamin B12 deficiency compared to total gastrectomy.[13, 26] Moreover, the nutritional outcomes of the DTR group were similar to those of the EG group in this study. Therefore, DTR can be a favorable option after laparoscopic proximal gastrectomy.

QOL is another important outcome of proximal gastrectomy as a function-preserving gastrectomy. To date, a limited number of studies have been reported, and none have compared EG and the DTR so far. In previous studies, EG was associated with a better QOL in terms of weight loss and diarrhea compared to total gastrectomy.[27, 28] On the other hand, the EG method, including jejunal interposition and doubletract reconstruction, had no long-term benefit in QOL compared with total gastrectomy.[13,29] In this study, we expected some differences in the reflux and dysphagia subscales related to anastomotic complications; however, there was no significant difference between the two groups. It is possible that some patients with reflux esophagitis in the EG group only had mild symptoms, or their symptoms were well controlled by a proton-pump inhibitor. In many previous studies, the endoscopic findings of reflux esophagitis were not consistent with the patients' symptoms.[30] Regarding anastomotic stenosis, the treatment of anastomotic stenosis is generally performed within 1-year after surgery, and the symptoms might improve by the time point of the QOL survey (1 year postoperatively). Moreover, it should be considered that approximately half of the patients completed the QOL questionnaire and non-responder bias cannot be excluded. Therefore, additional large-scale prospective studies are needed to confirm the difference of QOL between the EG and DTR groups.

There are several limitations to this study. First, data collection was from two institutions, and some surgical procedures and postoperative management might have been different between the institutes. Second, this study was retrospectively performed, and there were some missing data in nutritional parameters and QOL answers. The laboratory tests for iron deficiency anemia and vitamin B12 deficiency 
have been conducted routinely since 2015 and selectively performed in high-risk patients before 2015 . Thus, laboratory findings for iron deficiency anemia were collected in $100(97.1 \%)$ of 103 patients and those for vitamin B12 deficiency in 85 (82.5\%) patients. The QOL data were collected by a simple survey request in the outpatient clinic, and only 58 patients completed the answers both preoperatively and 1year postoperatively. Therefore, this study might include selection bias.

\section{Conclusion}

EG had higher incidence of esophageal reflux and similar nutritional outcomes and QOL compared with the double-tract reconstruction after laparoscopic proximal gastrectomy for early proximal gastric cancer. Further large-scale research is needed to evaluate the long-term functional outcomes of EG and the DTR.

\section{Abbreviations}

EG: Esophagogastrostomy

DTR: Double tract reconstruction

QOL: Quality of life

ASA: American Society of Anesthesiologists

WHO: World Health Organization

TNM: Tumor-Node-Metastasis

LA: Los Angeles

EORTC: European Organization for Research and Treatment of Cancer

ANOVA: one-way analysis of variance

BMI: body mass index

\section{Declarations}

\section{Availability of data and materials}

The datasets used and /or analyzed are available from the corresponding authors on reasonable request.

\section{Acknowledgments}

None

\section{Funding}


This work was supported by a grant of the National Cancer Center (No. 1910242).

\section{Author information}

\section{Affliations}

Center for Gastric Cancer, National Cancer Center, 323 Ilsan-ro, Ilsandong-gu, Goyang, 10408, Republic of Korea

Bang Wool Eom, Hong Man Yoon, Keun Won Ryu, Young-Woo Kim

Department of Surgery, School of Medicine, Kyungpook National University, 807 Hoguk-ro, Daegu, 41404, Republic of Korea

Ji Yeon Park, Ki Bum Park, Oh Kyoung Kwon

Department of Cancer Control and Population Health, Graduate School of Cancer Science and Policy, 323 Ilsan-ro, Ilsandong-gu, Goyang, 10408, Republic of Korea

Young-Woo Kim

\section{Contributions}

Bang Wool Eom and Ji Yeon Park contributed to the study design. Bang Wool Eom, Hong Man Yoon, Keun Won Ryu, Young-Woo Kim, Ji Yeon Park, Ki Bum Park, and Oh Kyoung Kwon contributed to the data collection. Bang Wool Eom contributed to the data analysis and manuscript writing. All authors read and edited the initial manuscript and proved the final manuscript.

\section{Corresponding author}

Correspondence to Ji Yeon Park

\section{Ethics declarations}

\section{Ethics approval and consent to participate}

The Institutional Review Board of the National Cancer Center (NCC2017-0120) and the Kyungpook National University Chilgok Hospital (2019-07-004) approved this study and waived the need for patient informed consent due to the retrospective nature of the study.

\section{Consent for publication}

Not applicable.

\section{Conflict of interest}


The authors (Drs. Bang Wool Eom, Ji Yeon Park, Ki Bum Park, Hong Man Yoon, Oh Kyoung Kwon, Keun Won Ryu, and Young-Woo Kim) have no conflicts of interest or financial ties to disclosure.

\section{References}

1. Borch K, Jonsson B, Tarpila E, Franzen T, Berglund J, Kullman E, Franzen L: Changing pattern of histological type, location, stage and outcome of surgical treatment of gastric carcinoma. Br J Surg 2000, 87:618-626.

2. Botterweck AA, Schouten LJ, Volovics A, Dorant E, van Den Brandt PA: Trends in incidence of adenocarcinoma of the oesophagus and gastric cardia in ten European countries.Int $\mathrm{J}$ Epidemiol 2000, 29:645-654.

3. Korean Gastric Cancer Association Nationwide Survey on Gastric Cancer in 2014.J Gastric Cancer 2016, 16:131-140.

4. Wen L, Chen XZ, Wu B, Chen XL, Wang L, Yang K, Zhang B, Chen ZX, Chen JP, Zhou ZG, et al: Total vs. proximal gastrectomy for proximal gastric cancer: a systematic review and metaanalysis. Hepatogastroenterology 2012, 59:633-640.

5. Ahn HS, Lee HJ, Yoo MW, Jeong SH, Park DJ, Kim HH, Kim WH, Lee KU, Yang HK: Changes in clinicopathological features and survival after gastrectomy for gastric cancer over a 20-year period.Br J Surg 2011, 98:255-260.

6. Nashimoto A, Akazawa K, Isobe Y, Miyashiro I, Katai H, Kodera Y, Tsujitani S, Seto Y, Furukawa H, Oda I, et al: Gastric cancer treated in 2002 in Japan: 2009 annual report of the JGCA nationwide registry.Gastric Cancer 2013, 16:1-27.

7. Sakuramoto S, Yamashita K, Kikuchi S, Futawatari N, Katada N, Moriya H, Hirai K, Watanabe M: Clinical experience of laparoscopy-assisted proximal gastrectomy with Toupet-like partial fundoplication in early gastric cancer for preventing reflux esophagitis.J Am Coll Surg 2009, 209:344351.

8. Toyomasu Y, Ogata K, Suzuki M, Yanoma T, Kimura A, Kogure N, Yanai M, Ohno T, Mochiki E, Kuwano $\mathrm{H}$ : Restoration of gastrointestinal motility ameliorates nutritional deficiencies and body weight loss of patients who undergo laparoscopy-assisted proximal gastrectomy.Surg Endosc 2017, 31:13931401.

9. Kuroda S, Nishizaki M, Kikuchi S, Noma K, Tanabe S, Kagawa S, Shirakawa Y, Fujiwara T: DoubleFlap Technique as an Antireflux Procedure in Esophagogastrostomy after Proximal Gastrectomy.J Am Coll Surg 2016, 223:e7-e13.

10. Ahn SH, Jung do H, Son SY, Lee CM, Park do J, Kim HH: Laparoscopic double-tract proximal gastrectomy for proximal early gastric cancer.Gastric Cancer 2014, 17:562-570.

11. Nomura E, Lee SW, Kawai M, Yamazaki M, Nabeshima K, Nakamura K, Uchiyama K: Functional outcomes by reconstruction technique following laparoscopic proximal gastrectomy for gastric cancer: double tract versus jejunal interposition. World J Surg Oncol 2014, 12:20. 
12. Hayami M, Hiki N, Nunobe S, Mine S, Ohashi M, Kumagai K, Ida S, Watanabe M, Sano T, Yamaguchi T: Clinical Outcomes and Evaluation of Laparoscopic Proximal Gastrectomy with Double-Flap Technique for Early Gastric Cancer in the Upper Third of the Stomach.Ann Surg Oncol 2017, 24:16351642.

13. Park JY, Park KB, Kwon OK, Yu W: Comparison of laparoscopic proximal gastrectomy with doubletract reconstruction and laparoscopic total gastrectomy in terms of nutritional status or quality of life in early gastric cancer patients.Eur J Surg Oncol 2018, 44:1963-1970.

14. Inaba K, Satoh S, Ishida Y, Taniguchi K, Isogaki J, Kanaya S, Uyama I: Overlap method: novel intracorporeal esophagojejunostomy after laparoscopic total gastrectomy.J Am Coll Surg 2010, 211:e25-29.

15. Bosman FT CF, Hruban RH, Theise ND: WHO Classification of Tumours of the Digestive System. WHO; 2010.

16. Owens WD, Felts JA, Spitznagel EL, Jr.: ASA physical status classifications: a study of consistency of ratings.Anesthesiology 1978, 49:239-243.

17. TNM-Classification of Malignant Tumours. Eighth Edition. Chichester, West Sussex, UK; Hoboken, NJ: Wiley-Blackwell 2017.

18. Organization WH: Iron deficiency anaemia: assessment, prevention, and control. A guide for programme managers. In. Geneva: World Health Organization; 2001

19. Cook JD: Diagnosis and management of iron-deficiency anaemia.Best Pract Res Clin Haematol 2005, 18:319-332.

20. Hu Y, Kim HI, Hyung WJ, Song KJ, Lee JH, Kim YM, Noh SH: Vitamin B(12) deficiency after gastrectomy for gastric cancer: an analysis of clinical patterns and risk factors.Ann Surg 2013, 258:970-975.

21. Armstrong D: Endoscopic evaluation of gastro-esophageal reflux disease. Yale J Biol Med 1999, 72:93-100.

22. Vickery CW, Blazeby JM, Conroy T, Arraras J, Sezer O, Koller M, Rosemeyer D, Johnson CD, Alderson D: Development of an EORTC disease-specific quality of life module for use in patients with gastric cancer.Eur J Cancer 2001, 37:966-971.

23. Blazeby JM, Conroy T, Bottomley A, Vickery C, Arraras J, Sezer O, Moore J, Koller M, Turhal NS, Stuart $\mathrm{R}$, et al: Clinical and psychometric validation of a questionnaire module, the EORTC QLQ-STO 22, to assess quality of life in patients with gastric cancer.Eur J Cancer 2004, 40:2260-2268.

24. Fayers P AN, Bjordal K, Groenveld M, Curran D, Bottomley A: The EORTC QLQ-C30 scoring manual. Brussels, Belgium: European Organisation for Research and Treatment of Cancer; 2001.

25. Polkowski WP, Mielko J, Geca K, Rawicz-Pruszynski K, Cisel B, Kurylcio A, Skorzewska M: Proximal Gastric Resection with Posterior Esophago-Gastrostomy and Partial Neo-Fundoplication in the Treatment of Advanced Upper Gastric Carcinoma.Dig Surg 2019:1-10.

26. Cho M, Son T, Kim HI, Noh SH, Choi S, Seo WJ, Roh CK, Hyung WJ: Similar hematologic and nutritional outcomes after proximal gastrectomy with double-tract reconstruction in comparison to 
total gastrectomy for early upper gastric cancer.Surg Endosc 2018.

27. Takiguchi N, Takahashi M, Ikeda M, Inagawa S, Ueda S, Nobuoka T, Ota M, Iwasaki Y, Uchida N, Kodera Y, Nakada K: Long-term quality-of-life comparison of total gastrectomy and proximal gastrectomy by postgastrectomy syndrome assessment scale (PGSAS-45): a nationwide multiinstitutional study.Gastric Cancer 2015, 18:407-416.

28. Nishigori T, Okabe H, Tsunoda S, Shinohara H, Obama K, Hosogi H, Hisamori S, Miyazaki K, Nakayama T, Sakai Y: Superiority of laparoscopic proximal gastrectomy with hand-sewn esophagogastrostomy over total gastrectomy in improving postoperative body weight loss and quality of life.Surg Endosc 2017, 31:3664-3672.

29. Namikawa T, Oki T, Kitagawa H, Okabayashi T, Kobayashi M, Hanazaki K: Impact of jejunal pouch interposition reconstruction after proximal gastrectomy for early gastric cancer on quality of life: short- and long-term consequences.Am J Surg 2012, 204:203-209.

30. Ronkainen J, Aro P, Storskrubb T, Lind T, Bolling-Sternevald E, Junghard O, Talley NJ, Agreus L: Gastro-oesophageal reflux symptoms and health-related quality of life in the adult general population-the Kalixanda study.Aliment Pharmacol Ther 2006, 23:1725-1733.

\section{Tables}


Table 1

Patient demographic and pathological characteristics

\begin{tabular}{|c|c|c|c|c|}
\hline Factors & Subgroup & EG & DTR & $P$ \\
\hline & & $(n=45)(\%)$ & $(n=58)(\%)$ & \\
\hline Age (median, IQR)(yr) & & $63.0(53.5,72.5)$ & $65.5(52.8,72.0)$ & 0.833 \\
\hline \multirow[t]{2}{*}{ Sex } & Male & $33(73.3)$ & $47(81.0)$ & 0.475 \\
\hline & Female & $12(26.7)$ & $11(19.0)$ & \\
\hline $\mathrm{BMI}($ mean $\pm \mathrm{SD})\left(\mathrm{kg} / \mathrm{m}^{2}\right)$ & & $25.0 \pm 3.3$ & $23.5 \pm 3.1$ & 0.018 \\
\hline \multirow[t]{3}{*}{ ASA } & I & $13(28.9)$ & $21(36.2)$ & 0.131 \\
\hline & II & $29(64.4)$ & $37(63.8)$ & \\
\hline & III & $3(6.7)$ & $0(0)$ & \\
\hline Tumor size (median, IQR)(cm) & & $2.4(1.6,3.9))$ & $2.0(1.3,2.8)$ & 0.043 \\
\hline $\begin{array}{l}\text { Number of positive lymph node } \\
\text { (median, IQR) }\end{array}$ & & $0(0,0)$ & $0(0,0)$ & 0.996 \\
\hline $\begin{array}{l}\text { Number of harvested lymph node } \\
\text { (median, IQR) }\end{array}$ & & $26.0(20.5,36.5)$ & $34.0(24.6,40.3)$ & 0.022 \\
\hline \multirow[t]{2}{*}{ pT } & 1 & $38(84.4)$ & $58(100)$ & 0.02 \\
\hline & 2 & $7(15.6)$ & $0(0)$ & \\
\hline \multirow[t]{2}{*}{$\mathrm{pN}$} & 0 & $41(91.1)$ & $57(98.3)$ & 0.165 \\
\hline & 1 & $4(8.9)$ & $1(1.7)$ & \\
\hline $\begin{array}{l}\text { EG, esophagogastrostomy; DTR, d } \\
\text { index; ASA, American Society of Ar } \\
\text { interquartile range }\end{array}$ & $\begin{array}{l}\text {-tract recol } \\
\text { iesiologists }\end{array}$ & $\begin{array}{l}\text { ruction; SD, standa } \\
\text { nysical status clas }\end{array}$ & $\begin{array}{l}\text { deviation; BMI, bo } \\
\text { cation system; IQF }\end{array}$ & mass \\
\hline
\end{tabular}


Table 2

Anastomotic complications in endoscopic evaluation within 1 year after surgery

\begin{tabular}{|lllll|}
\hline & & $\begin{array}{l}\text { EG } \\
(\mathbf{n = 4 5 )}\end{array}$ & $\begin{array}{l}\text { DTR } \\
(\mathbf{n = 5 8 )}\end{array}$ & p-value \\
\hline Esophageal reflux & Absence & $37(82.2)$ & $56(96.6)$ & 0.041 \\
\hline & LA-A* & $3(6.7)$ & $1(1.7)$ & \\
\hline LA-B/C* & $5(11.1)$ & $1(1.7)$ & \\
\hline Anastomotic stricture & No & $34(75.6)$ & $53(91.4)$ & 0.068 \\
\hline & Mild & $6(13.3)$ & $2(3.4)$ & \\
\hline & Severe & $5(11.1)$ & $3(5.2)$ & \\
\hline EG, esophagogastrostomy; DTR, double-tract reconstruction & \\
\hline *, Los Angeles classification & & & \\
\hline
\end{tabular}

Table 3

Incidence of nutritional deficiencies within 1 year after surgery

\begin{tabular}{|lllll|}
\hline & & EG & DTR & p-value \\
\hline Iron deficiency anemia & Absence & $38(90.5)$ & $51(87.9)$ & 0.757 \\
\hline & Present & $4(9.5)$ & $7(12.1)$ & \\
\hline Vitamine B12 deficiency & Absence & $30(85.7)$ & $41(82.0)$ & 0.771 \\
\hline & Present & $5(14.3)$ & $9(18.0)$ & \\
\hline EG, esophagogastrostomy; DTR, double-tract reconstruction & \\
\hline
\end{tabular}


Table 4

Differences between the preoperative and postoperative quality of life assessed by the EORTC QLQ STO-22

\begin{tabular}{|llll|}
\hline Subscale & EG & DTR & $P$ \\
\cline { 2 - 4 } & $(\mathbf{n = 2 2})$ & $(\mathbf{n = 3 6})$ & (Mann-Whitney $\mathbf{)}$ \\
\cline { 2 - 4 } & median (IQR) & median (IQR) & \\
\hline Dysphagia & $11.1(0,22.2)$ & $11.1(-8.3,22.2)$ & 0.396 \\
\hline Pain & $8.3(0,18.8)$ & $8.3(0,22.9)$ & 0.520 \\
\hline Reflux & $11.1(0,25.0)$ & $0(0,19.4)$ & 0.350 \\
\hline Eating restrictions & $20.8(6.2,25.0)$ & $16.7(2.1,25.0)$ & 0.728 \\
\hline Anxiety & $16.7(0,33.3)$ & $0(0,11.1)$ & 0.103 \\
\hline Dry mouth & $0(0,0)$ & $0(0,33.3)$ & 0.280 \\
\hline Taste & $0(0,33.3)$ & $0(0,33.3)$ & 0.412 \\
\hline Body image & $0(0,33.3)$ & $33.3(0,33.3)$ & 0.072 \\
\hline Hair loss & $0(0,0)$ & $0(0,0)$ & 0.355 \\
\hline EG, esophagogastrostomy; DTR, double-tract reconstruction \\
\hline
\end{tabular}

Figures 

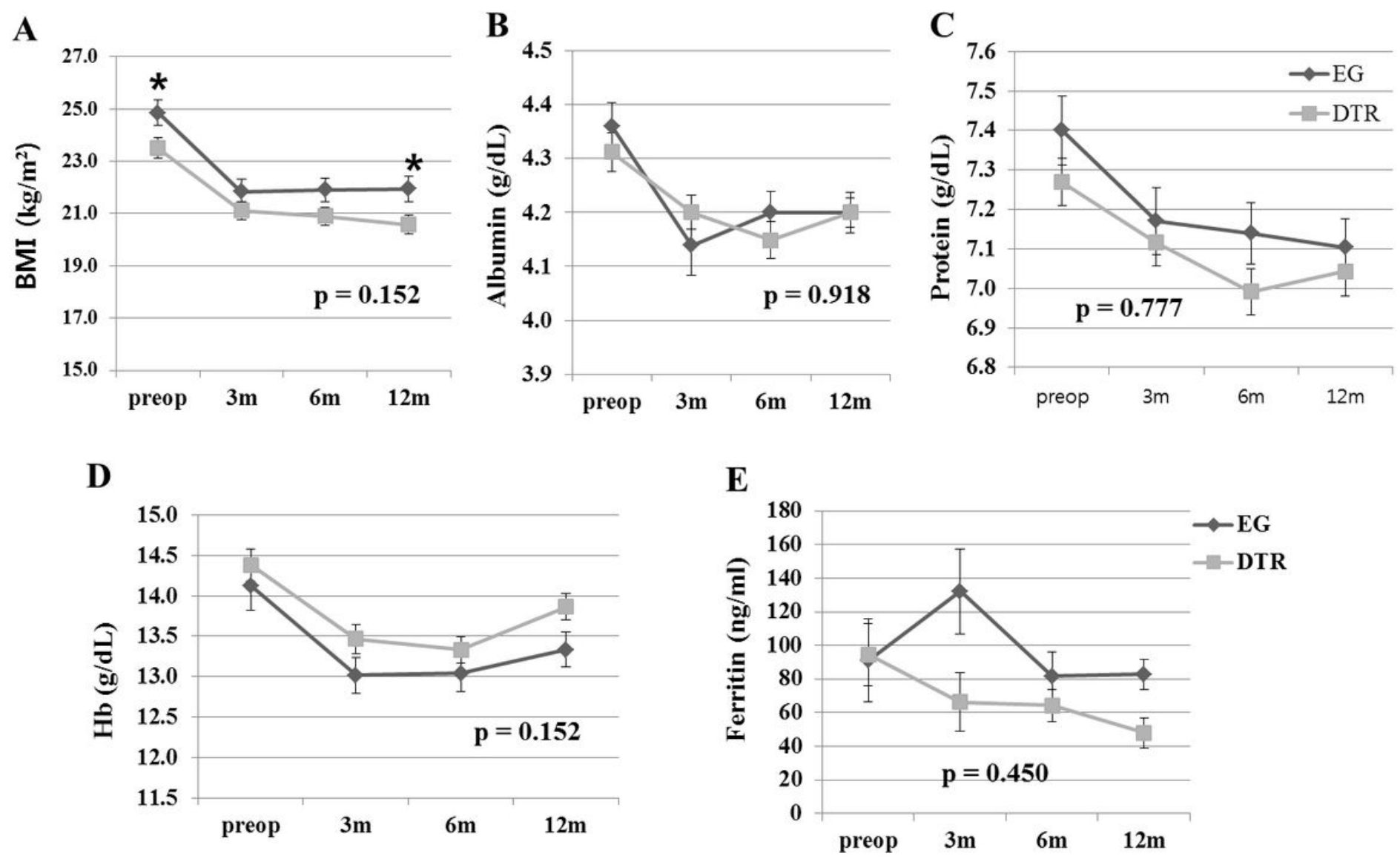

\section{Figure 1}

Comparison of changes in nutritional parameters between the esophagogastrostomy and double tract reconstruction after laparoscopic proximal gastrectomy. Levels of A body mass index, B serum albumin, $\mathrm{C}$ serum protein, $\mathrm{D}$ serum hemoglobin, and $\mathrm{E}$ serum ferritin. $\mathrm{BMI}$, body mass index; $\mathrm{Hb}$, hemoglobin; $\mathrm{EG}$ esophagogastrostomy; DTR, double-tract reconstruction *, significantly different at a specific time point $(p<0.05)$ 